\title{
Detection of scalar gravitational waves with a hollow spherical antenna
}

\author{
E. Coccia, F. Fucito, and M. Salvino \\ Dipartimento di Fisica, Università di Roma "Tor Vergata"' and INFN Sezione di Roma Tor Vergata, Via Ricerca Scientifica 1, \\ 00133 Roma, Italy \\ J. A. Lobo \\ Departament de Física Fonamental, Universitat de Barcelona, Spain
}

(Received 20 October 1999; published 21 July 2000)

\begin{abstract}
We study the response and cross sections for the absorption of GW energy generated in a Jordan-BransDicke theory by a resonant mass detector shaped as a hollow sphere. As a source of the GW we take a binary system in the Newtonian approximation. For masses of the stars of the order of the solar mass, the emitted GW sweeps a range of frequencies which include the first resonant mode of the detector.
\end{abstract}

PACS number(s): 04.30.Db

\section{INTRODUCTION}

It seems reasonable to predict that the new gravitational wave $(\mathrm{GW})$ detectors now under construction, once they are operating at the maximum of their sensitivity, will be able to detect GWs. Will it be possible to use these future measurements to try to gain information on which is the theory of gravity at low energies? There is no particular reason, in fact, why a GW must be of spin 2 . In reality, many theories of gravity can be built which contain scalars and vectors. These theories are mathematically well founded. String theory, in particular, is believed to be consistent also as a quantum description of gravity. The predictions of these theories must then be checked against available experimental data. This forces the couplings and masses present in the Lagrangian to take values in well-defined domains. See [1] for a more detailed exposition. Once detected, one can also attempt to use GWs as a means to further constrain this picture. It seems to us relevant to try to develop the theory to the point where it can profit from new experimental insights. For these reasons we have analyzed in great detail in Ref. [2] — see also [3,4] the interaction and cross section of a massive elastic sphere with scalar waves.

An appealing variant of the massive sphere is a hollow sphere [5]. The latter has the remarkable property that it enables the detector to monitor GW signals in a significantly lower frequency range - down to about $200 \mathrm{~Hz}$ - than its massive counterpart for comparable sphere masses. This can be considered a positive advantage for a future worldwide network of GW detectors, as the sensitivity range of such antennas overlaps with that of the large scale interferometers, now in a rather advanced state of construction [6,7]. In this paper we study the response of such a detector to the GW energy emitted by a binary system constituted of stars of masses of the order of the solar mass. A simple formula for the GW energy can be obtained in the Newtonian approximation whose region of validity encompasses emitted frequencies of the order of the first resonant mode of the detector under study.

A hollow sphere obviously has the same symmetry as the massive one, so the general structure of its normal modes of vibration is very similar in both [5]. In particular, the hollow sphere is very well adapted to sense and monitor the presence of scalar modes in the incoming GW signal. This paper reports on the results of an extension of the analysis of the response of a hollow sphere to include scalar excitations. The methodology largely follows the pattern of Ref. [2]. So in Sec. II we briefly review the normal mode algebra of the hollow sphere; then in Sec. III we calculate the scalar cross sections for the absorption of GW energy in scalar modes, and in Sec. IV we assess the detectability of a few interesting sources on the assumption that they behave as Jordan-BransDicke emitters $[8,9]$ of GWs. Finally, Sec. V is devoted to a summary of conclusions.

\section{REVIEW OF HOLLOW SPHERE NORMAL MODES}

This section contains some review material which is included essentially to fix the notation and to ease the reading of the ensuing sections. The notation will be that of Ref. [5]. The eigenmode equation for a three-dimensional elastic solid is the following:

$$
\nabla^{2} \mathbf{s}+(1+\lambda / \mu) \nabla(\nabla \cdot \mathbf{s})=-k^{2} \mathbf{s} \quad\left(k^{2} \equiv \varrho \omega^{2} / \mu\right),
$$

as described in standard textbooks, such as $[10,11]$. The equation must be solved subject to the boundary conditions that the solid is to be free from tensions and/or tractions. In the case of a hollow sphere, we have two boundaries given by the outer and the inner surfaces of the solid itself. We use the notation $a$ for the inner radius and $R$ for the outer radius. The boundary conditions are thus expressed by

$$
\sigma_{i j} n_{j}=0 \text { at } r=R \text { and at } r=a \quad(R \geqslant a \geqslant 0),
$$

where $\sigma_{i j}$ is the stress tensor, and is given by [11]

$$
\sigma_{i j}=\lambda u_{k, k} \delta_{i j}+2 \mu u_{(i, j)},
$$

with $\lambda$ and $\mu$ the material's Lamé coefficients, and $\mathbf{n}$ the unit, outward pointing normal vector.

The general solution to Eq. (2.1) is a linear superposition of a longitudinal vector field and two transverse vector fields, i.e., 


$$
\mathbf{s}(r, \vartheta, \phi)=\frac{C_{1}}{q} \mathbf{s}_{1}+\frac{C_{\mathrm{t}}}{k} \mathbf{s}_{\mathrm{t}}+C_{\mathrm{t}^{\prime}} \mathbf{s}_{\mathrm{t}^{\prime}},
$$

where $C_{1}, C_{\mathrm{t}}$, and $C_{\mathrm{t}^{\prime}}$ are constant coefficients, and

$$
\begin{aligned}
\mathbf{s}_{\mathrm{l}}(r, \vartheta, \phi)= & \frac{d h_{l}(q r, E)}{d r} Y_{l m} \mathbf{n}-\frac{h_{l}(q r, E)}{r} i \mathbf{n} \times \mathbf{L} Y_{l m}, \\
\mathbf{s}_{\mathrm{t}}(r, \vartheta, \phi)= & -l(l+1) \frac{h_{l}(k r, F)}{r} Y_{l m} \mathbf{n} \\
& +\left[\frac{h_{l}(k r, F)}{r}+\frac{d h_{l}(k r, F)}{d r}\right] i \mathbf{n} \times \mathbf{L} Y_{l m}
\end{aligned}
$$

$$
\mathbf{s}_{\mathrm{t}^{\prime}}(r, \vartheta, \phi)=h_{l}(k r, F) i \mathbf{L} Y_{l m},
$$

with $E$ and $F$ also arbitrary constants:

$$
q^{2} \equiv k^{2} \frac{\mu}{\lambda+\mu}=\frac{\varrho_{0} \omega^{2}}{\lambda+\mu}
$$

and

$$
h_{l}(z, A) \equiv j_{l}(z)+A y_{l}(z) .
$$

Here $j_{l}, y_{l}$ are spherical Bessel functions [12]:

$$
\begin{aligned}
& j_{l}(z)=z^{l}\left(-\frac{1}{z} \frac{d}{d z}\right)^{l} \frac{\sin z}{z}, \\
& y_{l}(z)=-z^{l}\left(-\frac{1}{z} \frac{d}{d z}\right)^{l} \frac{\cos z}{z} .
\end{aligned}
$$

Finally, $\mathbf{L}$ is the angular momentum operator:

$$
\mathbf{L} \equiv-i \mathbf{x} \times \nabla
$$

The boundary conditions (2.2) must now be imposed on the generic solution to Eq. (2.1). After some rather heavy algebra it is finally found that there are two families of eigenmodes, the toroidal (purely rotational) and the spheroidal. Only the latter couple to GWs [13], so we shall be interested exclusively in them. The form of the associated wave functions is

$$
\mathbf{s}_{n l m}^{S}(r, \vartheta, \phi)=A_{n l}(r) Y_{l m}(\vartheta, \phi) \mathbf{n}-B_{n l}(r) i \mathbf{n} \times \mathbf{L} Y_{l m}(\vartheta, \phi),
$$

where the radial functions $A_{n l}(r)$ and $B_{n l}(r)$ have rather complicated expressions:

$$
\begin{aligned}
A_{n l}(r)= & C_{n l}\left[\frac{1}{q_{n l}^{S}} \frac{d}{d r} j_{l}\left(q_{n l}^{S} r\right)-l(l+1) K_{n l} \frac{j_{l}\left(k_{n l}^{S} r\right)}{k_{n l}^{S} r}\right. \\
& \left.+D_{n l} \frac{1}{q_{n l}^{S}} \frac{d}{d r} y_{l}\left(q_{n l}^{S} r\right)-l(l+1) \widetilde{D}_{n l} \frac{y_{l}\left(k_{n l}^{S} r\right)}{k_{n l}^{S} r}\right],
\end{aligned}
$$

$$
\begin{aligned}
B_{n l}(r)= & C_{n l}\left[\frac{j_{l}\left(q_{n l}^{S} r\right)}{q_{n l}^{S} r}-K_{n l} \frac{1}{k_{n l}^{S} r} \frac{d}{d r}\left\{r j_{l}\left(k_{n l}^{S} r\right)\right\}\right. \\
& \left.+D_{n l} \frac{y_{l}\left(q_{n l}^{S} r\right)}{q_{n l}^{S} r}-\widetilde{D}_{n l} \frac{1}{k_{n l}^{S} r} \frac{d}{d r}\left\{r y_{l}\left(k_{n l}^{S} r\right)\right\}\right] .
\end{aligned}
$$

Here $k_{n l}^{S} R$ and $q_{n l}^{S} R$ are dimensionless eigenvalues, and they are the solution to a rather complicated algebraic equation for the frequencies $\omega=\omega_{n l}$ in Eq. (2.1)—see [5] for details. In Eqs. (2.11a) and (2.11b) we have set

$$
K_{n l} \equiv \frac{C_{\mathrm{t}} q_{n l}^{S}}{C_{1} k_{n l}^{S}}, \quad D_{n l} \equiv \frac{q_{n l}^{S}}{k_{n l}^{S}} E, \quad \widetilde{D}_{n l} \equiv \frac{C_{\mathrm{t}} F q_{n l}^{S}}{C_{1} k_{n l}^{S}},
$$

and introduced the normalization constant $C_{n l}$, which is fixed by the orthogonality properties

$\int_{V}\left(\mathbf{s}_{n^{\prime} l^{\prime} m^{\prime}}^{S}\right)^{*} \cdot\left(\mathbf{s}_{n l m}^{S}\right) \varrho_{0} d^{3} x=M \delta_{n n^{\prime}} \delta_{l l^{\prime}} \delta_{m m^{\prime}}$,

where $M$ is the mass of the hollow sphere:

$$
M=\frac{4 \pi}{3} \varrho_{0} R^{3}\left(1-\mathrm{s}^{3}\right), \quad \varsigma \equiv \frac{a}{R} \leqslant 1
$$

Equation (2.13) fixes the value of $C_{n l}$ through the radial integral

$$
\int_{\varsigma R}^{R}\left[A_{n l}^{2}(r)+l(l+1) B_{n l}^{2}(r)\right] r^{2} d r=\frac{4 \pi}{3} \varrho_{0}\left(1-\varsigma^{3}\right) R^{3},
$$

as can be easily verified by suitable manipulation of Eq. (2.10) and the well-known properties of angular momentum operators and spherical harmonics. We shall later specify the values of the different parameters appearing in the above expressions as required in each particular case which will in due course be considered.

\section{ABSORPTION CROSS SECTIONS}

As seen in Ref. [3], a scalar-tensor theory of GWs such as Jordan-Brans-Dicke (JBD) predicts the excitation of the sphere's monopole modes as well as the $m=0$ quadrupole modes. In order to calculate the energy absorbed by the detector according to that theory it is necessary to calculate the energy deposited by the wave in those modes, and this in turn requires that we solve the elasticity equation with the GW driving term included in its right hand side. The result of such a calculation was presented in full generality in Ref. [3], and is directly applicable here because the structure of the oscillation eigenmodes of a hollow sphere is equal to that of a massive sphere-only the explicit form of the wave functions needs to be changed. We thus have

$$
E_{\mathrm{osc}}\left(\omega_{n l}\right)=\frac{1}{2} M b_{n l}^{2} \sum_{m=-l}^{l}\left|G^{(l m)}\left(\omega_{n l}\right)\right|^{2},
$$


where $G^{(l m)}\left(\omega_{n l}\right)$ is the Fourier amplitude of the corresponding incoming $\mathrm{GW}$ mode,

$$
\begin{aligned}
& b_{n 0}=-\frac{\varrho_{0}}{M} \int_{a}^{R} A_{n 0}(r) r^{3} d r, \\
& b_{n 2}=-\frac{\varrho_{0}}{M} \int_{a}^{R}\left[A_{n 2}(r)+3 B_{n 2}(r)\right] r^{3} d r,
\end{aligned}
$$

for monopole and quadrupole modes, respectively, and $A_{n l}(r)$ and $B_{n l}(r)$ are given by Eqs. (2.11). Explicit calculation yields

$$
\begin{aligned}
& \frac{b_{n 0}}{R}=\frac{3}{4 \pi} \frac{C_{n 0}}{1-s^{3}}\left[\Lambda(R)-\varsigma^{3} \Lambda(a)\right], \\
& \frac{b_{n 2}}{R}=\frac{3}{4 \pi} \frac{C_{n 2}}{1-\varsigma^{3}}\left[\Sigma(R)-\varsigma^{3} \Sigma(a)\right],
\end{aligned}
$$

with

$$
\begin{aligned}
\Lambda(z) \equiv & \frac{j_{2}\left(q_{n 0} z\right)}{q_{n 0} R}+D_{n 0} \frac{y_{2}\left(q_{n 0} z\right)}{q_{n 0} R}, \\
\Sigma(z) \equiv & \frac{j_{2}\left(q_{n 2} z\right)}{q_{n 2} R}-3 K_{n 2} \frac{j_{2}\left(k_{n 2} z\right)}{k_{n 2} R} \\
& +D_{n 2} \frac{y_{2}\left(q_{n 2} z\right)}{q_{n 2} R}-3 \widetilde{D}_{n 2} \frac{y_{2}\left(k_{n 2} z\right)}{k_{n 2} R} .
\end{aligned}
$$

The absorption cross section, defined as the ratio of the absorbed energy to the incoming flux, can be calculated thanks to an optical theorem, as proved, e.g., by Weinberg [14]. According to that theorem, the absorption cross section for a signal of frequency $\omega$ close to $\omega_{N}$, say, the frequency of the detector mode excited by the incoming $\mathrm{GW}$, is given by the expression

$$
\sigma(\omega)=\frac{10 \pi \eta c^{2}}{\omega^{2}} \frac{\Gamma^{2} / 4}{\left(\omega-\omega_{N}\right)^{2}+\Gamma^{2} / 4}
$$

where $\Gamma$ is the linewidth of the mode-which can be arbitrarily small, as assumed in the previous section-and $\eta$ is the dimensionless ratio:

$$
\eta=\frac{\Gamma_{\mathrm{grav}}}{\Gamma}=\frac{1}{\Gamma} \frac{P_{G W}}{E_{\mathrm{osc}}},
$$

where $P_{G W}$ is the energy reemitted by the detector in the form of GWs as a consequence of its being set to oscillate by the incoming signal. In the following we will only consider the case $P_{G W}=P_{\text {scalar-tensor }}$ with $[3,2]$

$$
\begin{aligned}
P_{\text {scalar-tensor }}= & \frac{2 G \omega^{6}}{5 c^{5}\left(2 \Omega_{B D}+3\right)}\left[\left|Q_{k k}(\omega)\right|^{2}\right. \\
& \left.+\frac{1}{3} Q_{i j}^{*}(\omega) Q_{i j}(\omega)\right],
\end{aligned}
$$

where $Q_{i j}(\omega)$ is the quadrupole moment of the hollow sphere,

$$
Q_{i j}(\omega)=\int_{\text {antenna }} x_{i} x_{j} \varrho(\mathbf{x}, \omega) d^{3} x
$$

and $\Omega_{B D}$ is the Brans-Dicke parameter.

\section{SCALAR-TENSOR CROSS SECTIONS}

Explicit calculation shows that $P_{\text {scalar-tensor }}$ is made up of two contributions:

$$
P_{\text {scalar-tensor }}=P_{00}+P_{20}
$$

where $P_{00}$ is the scalar or monopole contribution to the emitted power, while $P_{20}$ comes from the central quadrupole mode which, as discussed in [2,3], is excited together with monopole in JBD theory. One must, however, recall that monopole and quadrupole modes of the sphere happen at different frequencies, so that cross sections for them only make sense if defined separately. More precisely,

$$
\begin{aligned}
& \sigma_{n 0}(\omega)=\frac{10 \pi \eta_{n 0} c^{2}}{\omega^{2}} \frac{\Gamma_{n 0}^{2} / 4}{\left(\omega-\omega_{n 0}\right)^{2}+\Gamma_{n 0}^{2} / 4}, \\
& \sigma_{n 2}(\omega)=\frac{10 \pi \eta_{n 2} c^{2}}{\omega^{2}} \frac{\Gamma_{n 2}^{2} / 4}{\left(\omega-\omega_{n 2}\right)^{2}+\Gamma_{n 2}^{2} / 4},
\end{aligned}
$$

where $\eta_{n 0}$ and $\eta_{n 2}$ are defined like in Eq. (3.6), with all terms referring to the corresponding modes. After some algebra one finds that

$$
\begin{aligned}
& \sigma_{n 0}(\omega)=H_{n} \frac{G M v_{S}^{2}}{\left(\Omega_{B D}+2\right) c^{3}} \frac{\Gamma_{n 0}^{2} / 4}{\left(\omega-\omega_{n 0}\right)^{2}+\Gamma_{n 0}^{2} / 4}, \\
& \sigma_{n 2}(\omega)=F_{n} \frac{G M v_{S}^{2}}{\left(\Omega_{B D}+2\right) c^{3}} \frac{\Gamma_{n 2}^{2} / 4}{\left(\omega-\omega_{n 2}\right)^{2}+\Gamma_{n 2}^{2} / 4} .
\end{aligned}
$$

Here, we have defined the dimensionless quantities

$$
H_{n}=\frac{4 \pi^{2}}{9\left(1+\sigma_{P}\right)}\left(k_{n 0} b_{n 0}\right)^{2},
$$


TABLE I. Eigenvalues $k_{n 0}^{S} R$, relative weights $D_{n 0}$, and $H_{n}$ coefficients for a hollow sphere with Poisson ratio $\sigma_{P}=1 / 3$. Values are given for a few different thickness parameters $s$.

\begin{tabular}{|c|c|c|c|c|}
\hline$s$ & $n$ & $k_{n 0}^{S} R$ & $D_{n 0}$ & $H_{n}$ \\
\hline \multirow[t]{4}{*}{0.01} & 1 & 5.48738 & $-1.43328 \cdot 10^{-4}$ & 0.90929 \\
\hline & 1 & 12.2332 & $-1.59636 \cdot 10^{-3}$ & 0.14194 \\
\hline & 2 & 18.6321 & $-5.58961 \cdot 10^{-3}$ & 0.05926 \\
\hline & 4 & 24.9693 & -0.001279 & 0.03267 \\
\hline \multirow[t]{4}{*}{0.10} & 1 & 5.45410 & -0.014218 & 0.89530 \\
\hline & 1 & 11.9241 & -0.151377 & 0.15048 \\
\hline & 2 & 17.7277 & -0.479543 & 0.04922 \\
\hline & 4 & 23.5416 & -0.859885 & 0.04311 \\
\hline \multirow[t]{4}{*}{0.15} & 1 & 5.37709 & -0.045574 & 0.86076 \\
\hline & 2 & 11.3879 & -0.434591 & 0.17646 \\
\hline & 3 & 17.105 & -0.939629 & 0.05674 \\
\hline & 4 & 23.605 & -0.806574 & 0.05396 \\
\hline \multirow[t]{4}{*}{0.25} & 1 & 5.04842 & -0.179999 & 0.73727 \\
\hline & 2 & 10.6515 & -0.960417 & 0.30532 \\
\hline & 3 & 17.8193 & -0.425087 & 0.04275 \\
\hline & 4 & 25.8063 & 0.440100 & 0.06347 \\
\hline \multirow[t]{4}{*}{0.50} & 1 & 3.96914 & -0.631169 & 0.49429 \\
\hline & 2 & 13.2369 & 0.531684 & 0.58140 \\
\hline & 3 & 25.4531 & 0.245321 & 0.01728 \\
\hline & 4 & 37.9129 & 0.161117 & 0.07192 \\
\hline \multirow[t]{4}{*}{0.75} & 1 & 3.26524 & -0.901244 & 0.43070 \\
\hline & 2 & 25.3468 & 0.188845 & 0.66284 \\
\hline & 3 & 50.3718 & 0.093173 & 0.00341 \\
\hline & 4 & 75.469 & 0.061981 & 0.07480 \\
\hline \multirow[t]{4}{*}{0.90} & 1 & 2.98141 & -0.963552 & 0.42043 \\
\hline & 2 & 62.9027 & 0.067342 & 0.67689 \\
\hline & 3 & 125.699 & 0.033573 & 0.00047 \\
\hline & 4 & 188.519 & 0.022334 & 0.07538 \\
\hline
\end{tabular}

$$
F_{n}=\frac{8 \pi^{2}}{15\left(1+\sigma_{P}\right)}\left(k_{n 2} b_{n 2}\right)^{2},
$$

where $\sigma_{P}$ represents the sphere material's Poisson ratio (most often very close to a value of $1 / 3$ ), and the $b_{n l}$ are defined in Eq. (3.3); $v_{S}$ is the speed of sound in the material of the sphere.

In Tables I and II we give a few numerical values of the above cross section coefficients.

As already stressed in Ref. [5], one of the main advantages of a hollow sphere is that it enables one to reach good sensitivities at lower frequencies than a solid sphere. For example, a hollow sphere of the same material and mass as a solid one $(s=0)$ has eigenfrequencies which are smaller by

$$
\omega_{n l}(\mathrm{~s})=\omega_{n l}(\mathrm{~s}=0)\left(1-\mathrm{s}^{3}\right)^{1 / 3}
$$

for any mode indices $n$ and $l$. We now consider the detectability of JBD GWs coming from several interesting sources with a hollow sphere.
TABLE II. Eigenvalues $k_{n 2}^{S} R$, relative weights $K_{n 2}, D_{n 2}, \widetilde{D}_{n 2}$, and $F_{n}$ coefficients for a hollow sphere with Poisson ratio $\sigma_{P}$

\begin{tabular}{|c|c|c|c|c|c|c|}
\hline 5 & $n$ & $k_{n 2}^{S} R$ & $K_{n 2}$ & $D_{n 2}$ & $\widetilde{D}_{n 2}$ & $F_{n}$ \\
\hline \multirow[t]{3}{*}{0.10} & 1 & 2.63836 & 0.855799 & 0.000395 & -0.003142 & 2.94602 \\
\hline & 2 & 5.07358 & 0.751837 & 0.002351 & -0.018451 & 1.16934 \\
\hline & 3 & 10.96090 & 0.476073 & 0.009821 & -0.071685 & 0.02207 \\
\hline \multirow[t]{3}{*}{0.15} & 1 & 2.61161 & 0.796019 & 0.001174 & -0.009288 & 2.86913 \\
\hline & 2 & 5.02815 & 0.723984 & 0.007028 & -0.053849 & 1.24153 \\
\hline & 3 & 8.25809 & -2.010150 & -0.094986 & 0.672786 & 0.08113 \\
\hline \multirow[t]{4}{*}{0.25} & 1 & 2.49122 & 0.606536 & 0.003210 & -0.02494 & 2.55218 \\
\hline & 2 & 4.91223 & 0.647204 & 0.019483 & -0.13867 & 1.55022 \\
\hline & 3 & 8.24282 & -1.984426 & -0.126671 & 0.67506 & 0.05325 \\
\hline & 4 & 10.97725 & 0.432548 & -0.012194 & 0.02236 & 0.03503 \\
\hline \multirow[t]{4}{*}{0.50} & 1 & 1.94340 & 0.300212 & 0.003041 & -0.02268 & 1.61978 \\
\hline & 2 & 5.06453 & 0.745258 & 0.005133 & -0.02889 & 2.29572 \\
\hline & 3 & 10.11189 & 1.795862 & -1.697480 & 2.98276 & 0.19707 \\
\hline & 4 & 15.91970 & -1.632550 & -1.965780 & -0.30953 & 0.17108 \\
\hline \multirow[t]{4}{*}{0.75} & 1 & 1.44965 & 0.225040 & 0.001376 & -0.01017 & 1.15291 \\
\hline & 2 & 5.21599 & 0.910998 & -0.197532 & 0.40944 & 1.82276 \\
\hline & 3 & 13.93290 & 0.243382 & 0.748219 & -3.20130 & 1.08952 \\
\hline & 4 & 23.76319 & 0.550278 & -0.230203 & -0.81767 & 0.08114 \\
\hline \multirow[t]{4}{*}{0.90} & 1 & 1.26565 & 0.213082 & 0.001019 & -0.00755 & 1.03864 \\
\hline & 2 & 4.97703 & 0.939420 & -0.323067 & 0.52279 & 1.54106 \\
\hline & 3 & 31.86429 & 6.012680 & -0.259533 & 4.05274 & 1.46486 \\
\hline & 4 & 61.29948 & 0.205362 & -0.673148 & -1.04369 & 0.13470 \\
\hline
\end{tabular}
$=1 / 3$. Values are given for a few different thickness parameters $s$.

\section{DETECTABILITY OF JBD SIGNALS}

The values of the coefficients $F_{n}$ and $H_{n}$, together with the expressions (4.2) for the cross sections of the hollow sphere, can be used to estimate the maximum distances at which a coalescing compact binary system and a gravitational collapse event can be seen with such detector. We consider these in turn.

\section{A. Binary systems}

We consider as a source of GWs a binary system formed by two neutron stars, each of them with a mass of $m_{1}=m_{2}$ $=1.4 M_{\odot}$. The chirp mass corresponding to this system is $M_{c} \equiv\left(m_{1} m_{2}\right)^{3 / 5}\left(m_{1}+m_{2}\right)^{-1 / 5}=1.22 M_{\odot} \quad$ and $\quad \nu_{[5 \text { cycles }]}$ $=1270 \mathrm{~Hz} .{ }^{1}$ Repeating the analysis carried on in Sec. V of [16] we find a formula for the minimum distance at which a measurement can be performed given a certain signal to noise ratio (SNR), for a quantum limited detector

\footnotetext{
${ }^{1}$ The frequency $\nu_{[5 \text { cycles] }}$ is the one the system has when it is five cycles away from coalescence. It is considered that beyond this frequency disturbing effects distort the simple picture of a clean binary system—see [15] for further references.
} 


$$
\begin{aligned}
& r\left(\omega_{n 0}\right)=\left[\frac{5 \times 2^{1 / 3}}{32} \frac{1}{\left(\Omega_{B D}+2\right)\left(12 \Omega_{B D}+19\right)} \frac{G^{5 / 3} M_{c}^{5 / 3}}{c^{3}} \frac{M v_{S}^{2}}{\hbar \omega_{n 0}^{4 / 3} S N R} H_{n}\right]^{1 / 2}, \\
& r\left(\omega_{n 2}\right)=\left[\frac{5 \times 2^{1 / 3}}{192} \frac{1}{\left(\Omega_{B D}+2\right)\left(12 \Omega_{B D}+19\right)} \frac{G^{5 / 3} M_{c}^{5 / 3}}{c^{3}} \frac{M v_{S}^{2}}{\hbar \omega_{n 2}^{4 / 3} S N R} F_{n}\right]^{1 / 2} .
\end{aligned}
$$

For a CuAL sphere, the speed of sound is $v_{S}=4700$ $\mathrm{m} / \mathrm{sec}$. We report in Table III the maximum distances at which a JBD binary can be seen with a 100 ton hollow spherical detector, including the size of the sphere (diameter and thickness factor) for $\mathrm{SNR}=1$. The Brans-Dicke parameter $\Omega_{B D}$ has been given a value of 600 . This high value has as a consequence that only relatively nearby binaries can be scrutinized by means of their scalar radiation of GWs. A slight improvement in sensitivity is appreciated as the diameter increases in a fixed mass detector. Vacancies in the tables mean the corresponding frequencies are higher than $\nu_{[5 \text { cycles }]} \cdot$

\section{B. Gravitational collapse}

The signal associated with a gravitational collapse has recently been modeled, within JBD theory, as a short pulse of amplitude $b$, whose value can be estimated as [19]

$$
b \simeq 10^{-23}\left(\frac{500}{\Omega_{B D}}\right)\left(\frac{M_{*}}{M_{\odot}}\right)\left(\frac{10 \mathrm{Mpc}}{r}\right),
$$

where $M_{*}$ is the collapsing mass.

The minimum value of the Fourier transform of the amplitude of the scalar wave, for a quantum limited detector at unit signal-to-noise ratio, is given by [2]

$$
\left|b\left(\omega_{n l}\right)\right|_{\min }=\left(\frac{4 \hbar}{M v_{S}^{2} \omega_{n l} K_{n}}\right)^{1 / 2},
$$

where $K_{n}=2 H_{n}$ for the mode with $l=0$ and $K_{n}=F_{n} / 3$ for the mode with $l=2, m=0$.

The duration of the impulse, $\tau \approx 1 / f_{c}$, is much shorter than the decay time of the $n l$ mode, so that the relationship between $b$ and $b\left(\omega_{n l}\right)$ is

$$
b \approx\left|b\left(\omega_{n l}\right)\right| f_{c} \text { at frequency } \omega_{n l}=2 \pi f_{c}
$$

TABLE III. Eigenfrequencies, sizes, and distances at which coalescing binaries can be seen by monitoring of their emitted JBD GWs. Figures correspond to a 100 ton $\mathrm{CuAl}$ hollow sphere.

\begin{tabular}{lccccc}
\hline \hline$\varsigma$ & $\Phi(\mathrm{m})$ & $\nu_{10}(\mathrm{~Hz})$ & $\nu_{12}(\mathrm{~Hz})$ & $r\left(\nu_{10}\right)(\mathrm{kpc})$ & $r\left(\nu_{12}\right)(\mathrm{kpc})$ \\
\hline 0.00 & 2.94 & 1655 & 807 & - & 29.8 \\
0.25 & 2.96 & 1562 & 771 & - & 30.3 \\
0.50 & 3.08 & 1180 & 578 & 55 & 31.1 \\
0.75 & 3.5 & 845 & 375 & 64 & 33 \\
0.90 & 4.5 & 600 & 254 & 80 & 40 \\
\hline \hline
\end{tabular}

so that the minimum scalar wave amplitude detectable is

$$
|b|_{\min } \approx\left(\frac{4 \hbar \omega_{n l}}{\pi^{2} M v_{S}^{2} K_{n}}\right)^{1 / 2} .
$$

Let us now consider a hollow sphere made of molybdenum, for which the speed of sound is as high as $v_{S}=5600$ $\mathrm{m} / \mathrm{sec}$. For a given detector mass and diameter, Eq. (5.5) tells us which is the minimum signal detectable with such detector. For example, a solid sphere of $M=31$ tons and $1.8 \mathrm{~m}$ in diameter is sensitive down to $b_{\min }=1.5 \times 10^{-22}$. Equation (5.2) can then be inverted to find which is the maximum distance at which the source can be identified by the scalar waves it emits. Taking a reasonable value of $\Omega_{B D}=600$, one finds that $r\left(\nu_{10}\right) \approx 0.6 \mathrm{Mpc}$.

Like before, we report in Tables IV, V and VI the sensitivities of the detector and consequent maximum distance at which the source appears visible to the device for various values of the thickness parameter s. In Table $\mathrm{V}$ a detector of mass of 31 tons has been assumed for all thicknesses, and in Tables IV and VI a constant outer diameter of 3 and $1.8 \mathrm{~m}$ has been assumed in all cases.

\section{CONCLUSIONS}

In this paper we have explored the ability of a hollow sphere to sense the GWs emitted by radiating systems which obey the laws of Jordan-Brans-Dicke theory rather than those of general relativity. The difference between the predictions of both theories is in the degrees of freedom of the signal: while GR only predicts two polarization states-the usual + and $\times$ amplitudes - JBD predict a scalar amplitude and one more quadrupole amplitude $(m=0)$ in addition to the + and $\times$ amplitudes. All of these degrees of freedom can be measured with a spherical antenna which is thus able to distin-

TABLE IV. Eigenfrequencies, sizes and distances at which coalescing binaries can be seen by monitoring of their emitted JBD GWs. Figures correspond to a $3 \mathrm{~m}$ external diameter $\mathrm{CuAl}$ hollow sphere.

\begin{tabular}{lccccc}
\hline \hline & $M(\mathrm{ton})$ & $\nu_{10}(\mathrm{~Hz})$ & $\nu_{12}(\mathrm{~Hz})$ & $r\left(\nu_{10}\right)(\mathrm{kpc})$ & $r\left(\nu_{12}\right)(\mathrm{kpc})$ \\
\hline 0.00 & 105 & 1653 & 804 & - & 33 \\
0.25 & 103.4 & 1541 & 760 & - & 31 \\
0.50 & 92 & 1212 & 593 & 52 & 27.6 \\
0.75 & 60.7 & 997 & 442 & 44.8 & 23 \\
0.90 & 28.4 & 910 & 386 & 32 & 16.3 \\
\hline \hline
\end{tabular}


TABLE V. Eigenfrequencies, maximum sensitivities, and distances at which a gravitational collapse can be seen by monitoring the scalar GWs it emits. Figures correspond to a 31 ton Mb hollow sphere.

\begin{tabular}{lcccc}
\hline \hline$\varsigma$ & $\phi(\mathrm{m})$ & $\nu_{10}(\mathrm{~Hz})$ & $|b|_{\min }\left(10^{-22}\right)$ & $r\left(\nu_{10}\right)(\mathrm{Mpc})$ \\
\hline 0.00 & 1.80 & 3338 & 1.5 & 0.6 \\
0.25 & 1.82 & 3027 & 1.65 & 0.5 \\
0.50 & 1.88 & 2304 & 1.79 & 0.46 \\
0.75 & 2.16 & 1650 & 1.63 & 0.51 \\
0.90 & 2.78 & 1170 & 1.39 & 0.6 \\
\hline \hline
\end{tabular}

guish different theories of gravity. We have calculated the generic cross sections of the hollow spherical detectors for the first few monopole and quadrupole harmonics, which are relevant to the detection of JBD waves, then applied the results to the analysis of how efficient the detection of such waves coming from a coalescing compact binary system and a collapsing star, respectively, can be with a hollow spherical $\mathrm{GW}$ detector. Our numerical results are obtained at first order in the Newtonian approximation which is the correct regime, given the value of the first resonant mode of the detector and the typical masses of the stars making up the binary systems that we have used.

Reasoning the other way around, once actual measurements will be available, one will be able to restrict the parameters of JBD theory. Following the analysis in [19] we can in fact extract from Eq. (5.2) the value of $\Omega_{B D}$ as a function of the distance $r$ to obtain $\Omega_{B D} \geqslant 750(1 \mathrm{Mpc} / r)$. Analogous results can be obtained from Sec. V A. $\Omega_{B D}$ appears quadratically in Eqs. (5.1a) and (5.1b) and its expression as a function of $r$ is a little involved. Anyway the constraints one can deduce on $\Omega_{B D}$ are of the same order of magnitude of the ones deduced earlier.

In terms of the detector thickness it is seen that sensitivity appears to be, in absolute figures, quite independent of the detector geometry; i.e., the weakest detectable signal in the best experimental circumstances does not change in order of magnitude over the entire range of possible thickness parameter values. In other words, there is not much difference in
TABLE VI. Eigenfrequencies, maximum sensitivities and distances at which a gravitational collapse can be seen by monitoring the scalar GWs it emits. Figures correspond to a $1.8 \mathrm{~m}$ outer diameter $\mathrm{Mb}$ hollow sphere.

\begin{tabular}{lcccc}
\hline \hline s & $M$ (ton) & $\nu_{10}(\mathrm{~Hz})$ & $|b|_{\min }\left(10^{-22}\right)$ & $r\left(\nu_{10}\right)(\mathrm{Mpc})$ \\
\hline 0.00 & 31.0 & 3338 & 1.5 & 0.6 \\
0.25 & 30.52 & 3062 & 1.71 & 0.48 \\
0.50 & 27.12 & 2407 & 1.95 & 0.42 \\
0.75 & 17.92 & 1980 & 2.34 & 0.36 \\
0.90 & 8.4 & 1808 & 3.31 & 0.24 \\
\hline \hline
\end{tabular}

sensitivity between a hollow sphere and a massive one [2] for this kind of detection problem.

It is also possible to try to draw a comparison with the results attainable with a laser interferometer. L-shaped interferometers work in the dark fringe mode and thus detect most efficiently the difference of phases along the arms of the interferometer. Scalar modes "expand" or "contract" both arms in the same direction. It thus seems that in this interferometric configuration the only way to distinguish between scalar and tensor modes is to look at the sensitivity pattern in space of the detectors [17] which is a possibility clearly disfavored from the experimental point of view. Alternatively one could study different interferometric configurations in which the detection of the sum of phases has a sensitivity comparable to that of the standard setting [18]. Disregarding these concerns and considering the case in which the scalar modes affect only one arm of the interferometer in the case of VIRGO, it is possible to compute that $\mathrm{SNR}=1$ is attained for distances of the order of $r \simeq 70 \mathrm{kpc}$ for two neutron stars of 1.4 solar mass and $r \simeq 300 \mathrm{kpc}$ for two black holes of 10 solar mass [16].

As a final remark we would like to stress that one can reach considerably lower frequencies in the sensitivity range of a hollow sphere compared with those attainable with a massive one. Not surprisingly, the general facts described in Ref. [5] also survive in this more general framework of JBD theory.
[1] C.M. Will, Theory and Experiment in Gravitational Physics (Cambridge University Press, Cambridge, England, 1993).

[2] M. Bianchi, M. Brunetti, E. Coccia, F. Fucito, and J.A. Lobo, Phys. Rev. D 57, 4525 (1998).

[3] J.A. Lobo, Phys. Rev. D 52, 591 (1995).

[4] R.V. Wagoner and H.J. Paik, in Proceedings of the International Symposium on Experimental Gravitation (Accademia Nazionale dei Lincei, Rome, 1977).

[5] E. Coccia, V. Fafone, G. Frossati, J.A. Lobo, and J.A. Ortega, Phys. Rev. D 57, 2051 (1998).

[6] LIGO team, F.J. Raab, in Gravitational Wave Experiments, Proceedings of the First Edoardo Amaldi Conference, Frascati, 1994, edited by E. Coccia, G. Pizzella, and F. Ronga (World Scientific, Singapore, 1995).
[7] VIRGO Collaboration, A. Giazotto et al., in Gravitational Wave Experiments [6].

[8] P. Jordan, Z. Phys. 157, 112 (1959).

[9] C. Brans and R.H. Dicke, Phys. Rev. 124, 925 (1961).

[10] A.E.H. Love, A Treatise on the Mathematical Theory of Elasticity (Dover, New York, 1944).

[11] L.D. Landau and E.M. Lifshitz, Theory of Elasticity (Pergamon, New York, 1970).

[12] M. Abramowitz and I.A. Stegun, Handbook of Mathematical Functions (Dover, New York, 1972).

[13] M. Bianchi, E. Coccia, C.N. Colacino, V. Fafone, and F. Fucito, Class. Quantum Grav. 13, 2865 (1996).

[14] S. Weinberg, Gravitation and Cosmology (Wiley, New York, 1972). 
[15] E. Coccia and V. Fafone, Phys. Lett. A 213, 16 (1996).

[16] M. Brunetti, E. Coccia, V. Fafone, and F. Fucito, Phys. Rev. D 59, 044027 (1999).

[17] M. Maggiore and A. Nicolis, Phys. Rev. D 62, 024004 (2000).

[18] M.E. Tobar, T. Suzuki, and K. Kuroda, Phys. Rev. D 59,
102002 (1999)

[19] M. Shibata, K. Nakao, and T. Nakamura, Phys. Rev. D 50, 7304 (1994); M. Saijo, H. Shinkai, and K. Maeda, ibid. 56, 785 (1997) 